\section{Developing a sense of direction}

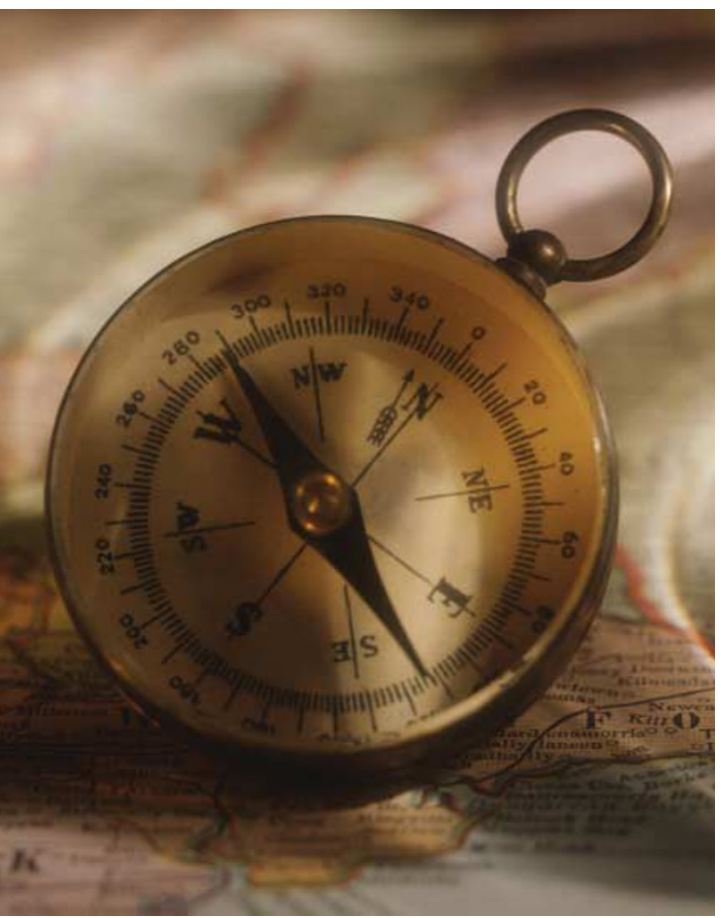

Metastasizing cancer cells seem to know exactly where they are going. But cancer cells don't normally express the chemokine receptors that are required for homing to target organs, so how do they develop this sense of direction? According to a report in Nature by Wilhelm Krek and colleagues, expression of the CXCR4 chemokine receptor is negatively regulated by the von Hippel-Lindau (VHL) tumour suppressor protein under normoxic conditions, and is induced by hypoxia-inducible factor (HIF) during hypoxic conditions.

The authors were comparing gene-expression profiles of VHL-deficient A498 renal carcinoma cells (RCCs) with A498 cells that were engineered to express a haemagglutinin-tagged VHL, and noticed that CXCR4 mRNA expression was strongly suppressed in the VHLexpressing cells. However, they went on to show that a mutant form of VHL — which prevents VHL from degrading HIF $1 \alpha$ - could not suppress CXCR4 expression, indicating that CXCR4 is directly regulated by HIF. CXCR4 mRNA accumulation in hypoxic renal epithelial cells occurs at a similar rate to GLUT3 - a known target of HIF - providing further evidence that CXCR4 is a hypoxia-inducible gene. So, there seems to be a link between loss of VHL function, hypoxia and overexpression of CXCR4.

They analysed the CXCR4 promoter and intron, and found four potential hypoxiaresponsive elements. Luciferase reporter assays that analysed this region showed a twofold increase in luciferase expression in response to hypoxia in VHL-positive human embryonic kidney cells. Co-transfection with wild-type HIF $1 \alpha$ enhanced reporter activity by tenfold and deletion analysis revealed an upsteam hypoxia-response element that was crucial for this HIF $1 \alpha$-inducible reporter activity. Electrophoretic mobility-shift assays showed that HIF $2 \alpha$ binds to this element, confirming that the CXCR4 promoter is targeted by HIF.

So, does expression of CXCR4 have an effect on cell migration? In transwell assays, increasing concentrations of stromal-derived factor $1 \alpha$ $(\mathrm{SDF} 1 \alpha)$ - the chemokine that is specific for CXCR4 - caused directed migration of A498 cells, and restoration of wild-type VHL abrogated this response. SDF1 $\alpha$ stimulation activates rapid accumulation of LIM kinase-1 and extracellular-signal-related kinases (ERKs) that control cell movement and proliferation. But, is inactivation of VHL and upregulation of CXCR4 occurring in vivo?

\section{MOUSE MODELS}

\section{Different strokes}

Lung cancer can come in one of two forms small cell and non small cell — that are initiated by different sets of genetic lesions and that have very different phenotypes. Mouse models have previously proven useful in identifying the changes that are required to induce non-small-cell lung cancer, and Anton Berns and colleagues have used the same approach to investigate the changes that cause small-cell lung cancer (SCLC). Interestingly, the changes are very different: instead of an activating mutation in Kras, both the Trp53 and $R b$ tumour-suppressor genes must be inactivated.

The authors investigated the role of $\mathrm{Rb}$ and p53 because they are frequently mutated in human SCLC. They generated mice with conditional alleles of the two genes, which could be inactivated in lung epithelial cells by administering Ad-Cre by either intratracheal injection or intubation. The lungs of these mice were examined after $2-5$ months and were found to contain areas of neoplasia in which both the $R b$ and $\operatorname{Tr} p 53$ alleles had been lost. These cells expressed two

neuroendocrine (NE) cell markers synaptophysin and the neural cell adhesion molecule 1 (Ncam1) - which indicates that they resemble SCLC, as SCLC is derived from cells that share the NE phenotype.

Mice that were not examined at this early stage were sacrificed when they became moribund. Three strains were investigated $\operatorname{Tr} p 53^{\mathrm{f} / \mathrm{f}} ; R b^{\mathrm{f} / \mathrm{f}}$ (in which all alleles were conditional), $\operatorname{Tr} p 53^{+/ / f} ; R b^{\mathrm{f} / \mathrm{f}}$ and $\operatorname{Tr} p 53^{\mathrm{ff/f}} ; R b^{+/ f}$ (in which one of the tumour suppressors retains a wild-type allele) — which differed in several respects. The $\operatorname{Tr} p 53^{\mathrm{f} / \mathrm{f}} ; R b^{\mathrm{f} / \mathrm{f}}$ mice had the shortest median tumour-free survival time of 210 days, with the $\operatorname{Tr} p 53^{+/ f} ; R b^{\mathrm{f} / \mathrm{f}}$ and $\operatorname{Tr} p 53^{\mathrm{f} / \mathrm{f}} ; R b^{+/ \mathrm{f}}$ mice remaining tumour free until 364 and 575 days, respectively. Tumours from the $\operatorname{Tr} p 53^{\mathrm{fff} ;} ; R b^{\mathrm{f} / \mathrm{f}}$ mice frequently metastasized to bone, brain, adrenal glands, ovaries and liver, whereas tumours from $\operatorname{Tr} p 53^{\mathrm{f} / f} ; R b^{+/ f}$ mice rarely did and from $\operatorname{Trp} 53^{+/ f} ; R b^{\mathrm{fff}}$ mice never did.

The primary tumour types that developed were also somewhat different. The $\operatorname{Trp} 53^{\mathrm{ff} / f} ; R b^{\mathrm{f} / \mathrm{f}}$ mice developed mostly SCLC - histological analysis confirmed their resemblance to human SCLC - but the other strains also developed some pulmonary adenocarcinomas. The longer survival time of the $\operatorname{Tr} p 53^{+/ f} ; R b^{\mathrm{f} / \mathrm{f}}$ and $\operatorname{Tr} p 53^{\mathrm{f} / \mathrm{f}} ; R b^{+/ \mathrm{f}}$ mice is probably related to the delay before the remaining wild-type allele is lost, as Southern blotting of the loci confirmed that loss of heterozygosity had occurred in all SCLC cases. Interestingly, this was not the case for pulmonary adenocarcinomas; PCR analysis of microdissected tissue from $\operatorname{Tr} p 53^{\mathrm{f} / \mathrm{f}} ; R b^{+/ \mathrm{f}}$ mice showed that loss of $\mathrm{Rb}$ was not required for development of the adenocarcinomas.

As well as NE markers, the achaete scute complex homologue-like 1 (ASCL1) transcription factor is frequently expressed in human SCLC, and it is thought to determine the onset of NE differentiation in lung epithelia and lung cancers with NE features. Indeed, the mouse SCLCs and their liver metastases generally stained positive for Ascl1 and the NE markers synaptophysin and Cgrp.

So, this mouse model closely resembles human SCLC, and shows that inactivation of both $\mathrm{p} 53$ and $\mathrm{Rb}$ are required for the initiation of SCLC. The applications of the 
